\title{
Resonance scattering across the superlattice barrier and the dimensional quantization
}

\author{
B. Pavlov $^{1}$, A. Yafyasov ${ }^{2}$ \\ ${ }^{1}$ New Zealand Institute of Advanced Studies, Auckland, New Zealand \\ ${ }^{2}$ Dept. of Solid State Electronics, St. Petersburg State University, St. Petersburg, Russia \\ yafyasov@gmail.com
}

\section{DOI 10.17586/2220-8054-2016-7-5-816-834}

\begin{abstract}
Carbon nano-cluster cathodes exhibit a low threshold electron emission, which is $2-3$ orders lower than on metals and semiconductors. We confirm the effect by direct experiments with graphene structures. We are suggesting a model based on the interference electrons wave function in 3D-space charge region of carbon structure interface with vacuum. The low-threshold emission is explained, in frames of the model, by the resonance properties of the barrier formed on the interface. Also in the following topics: interpretation of recent experimental findings for saturation of the field emission; local spectral analysis of multidimensional periodic lattices: dispersion via DN-map; examples of iso-energetic surfaces associated with solvable models of periodic lattice; Lagrangian version of the operator extension algorithm; solvable models of selected one-body spectral problems; quantum dot attached to the node of a quantum graph; a solvable model of a discrete lattice and spectral structure of a 1D superlattice via analytic perturbation procedure.
\end{abstract}

Keywords: periodic interface, carbon cover, Weyl-Titchmarsh function, resonance, field emission, DN-map.

Received: 23 August 2016

Revised: 1 September 2016

\section{An example: resonance concepts of the low-threshold field emission}

In numerous recent experiments, see for instance [1-7] extremely low-threshold field emission from metallic cathodes under carbon deposit was observed for electric fields $\left(10^{4}-10^{5} \mathrm{~V} / \mathrm{cm}\right)$. This is a surprisingly strong effect, because the field initiating a noticeable emission $\left(10^{-10}-10^{-9} A\right)$ from these materials is by $2-3$ orders of magnitude less than the field required for the field-emission from the traditional metals and semiconductors. Despite an obviously unusual nature of the effect, numerous authors, see for instance [5,6] attempted to explain the low-threshold phenomenon trivially with use of the classical Fowler-Nordheim techniques, based on enhancing of the field at the micro-protrusions. They assume that the local field $F_{s}$ near the emitting center is calculated as $F_{0}=\gamma F_{0}$, where $\gamma$ is the field enhancement coefficient, defined by the micro-geometry, and $F_{0}$ is the field of the equivalent flat capacitor. This completely classical explanation of the low-threshold emission phenomenon is not universal, and certainly non-valid for deposit, considered in our recent papers $[3,7]$ because the surface of the carbon flakes, obtained by the detonation synthesis, are perfectly smooth, see the flakes (see Fig. 1) under maximal magnifications.

with rare and relatively small protrusions. These protrusions are able to lower the threshold 5 -fold, while $10^{2}$ times lowering is observed in our experiments. We suggested in in $[3,7]$ an alternative explanation of the threshold lowering (field enhancement) based on the dimensional (size-) quantization in the under-surface spacecharge region. The classical Fowler-Nordheim techniques for calculating the transmission coefficient $T$ for simple rectangular potential barrier, see [8], gives an exponentially small value $T \approx e^{-q a}$ with $q=\sqrt{v-2 m E \hbar^{-2}}$ for the under-barrier tunneling with $v>>2 m E \hbar^{-2}$ and the width of the barrier equal to $a$. The resonance modification of the classical Fowler-Nordheim algorithm for calculating the transmission coefficient across a rectangular barrier, in presence of the energy levels of the size-quantization, meets some technical complications which can be avoided while substituting the rectangular barrier with delta-barrier supplied with an inner structure, attached to the barrier by Datta and Das-Sarma boundary condition, see $[9,10]$ for discussion of this phenomenological boundary condition and the derivation of it from the first principles in [11]. The program of resonance interpretation of the low-threshold field emission, based on zero-range model barrier with an inner structure, is developed in [7]. Where the zero-dimensional metal-carbon interface is substituted by the $1+0$ solvable model for electron transmission from the metallic cathode to vacuum through the $0 \mathrm{D}$ barrier, supplied with an inner structure, emulation of the discrete levels of the size quantization is interpreted as the Tamm surface state. Based on this model, we developed a resonance version of the classical Fowler-Nordheim machinery, considering the complex Tamm levels as resonances which serve as bridges helping electrons with Fermi energy to exit from the metallic cathode into 

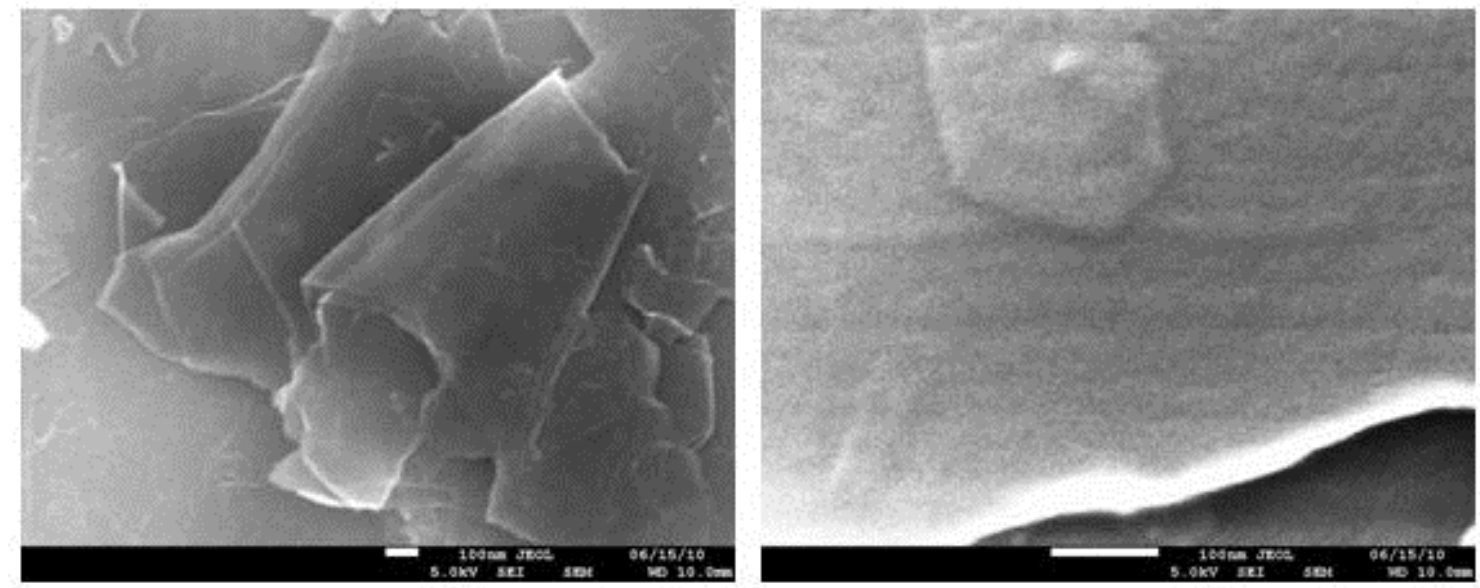

FIG. 1. Images of carbon Nano-clusters obtained via scanning electron microscope

vacuum across the carbon deposit. The role of the field enhancing factor in [7] was played by the small effective mass of electron in the carbon structure. Indeed, the field is measured by the steepness of the potential slope. But the effective steepness is calculated with respect to the De-Broghlie wavelength which is $m / m_{e}$ times bigger than the conventional De-Broghlie wavelength at the same energy. The corresponding formula for the transmission coefficient was derived for the general 1D model of the space-charge region, with complex discrete spectrum of Tamm surface levels. The presence of these resonance details in the barrier may result in much larger value of the transmission coefficient values $T$ for electrons with certain energy. In [7] we substitute the inner structure by a finite matrix, which is fit based on experimental data on size quantization, emulating the barrier barrier with inner structure by equivalent T-junction with the generalized Datta and Das Sarma boundary conditions, see [9-11]. The 1D model of the contact zone of the emitter in the form of a T-junction consisting of the cathode (1) $-\infty<x<0$, the vacuum (2) $0<x<\infty$, and the deposit (3) attached at the origin. The components $u_{s}, s=1,2,3$ of the wave-function of the electron satisfy on the intervals $(-\infty, 0),(0, \infty)$ the Schrödinger equations with appropriate potentials, see [7]:

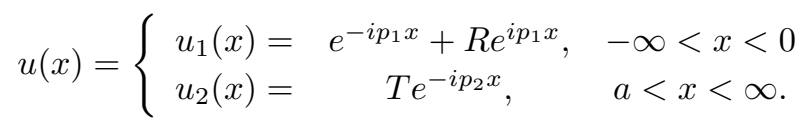

In [7], we model the component $u^{3}$ of the wave-function on the barrier by the finite vector and, correspondingly, substitute the barrier by a zero-range model with an inner structure, see [12,13], defined by the finite Hermitian matrix $A$. Interaction between components $u_{1}, u_{2}, u_{3}$ is defined via imposing boundary conditions onto the boundary data $u_{1}\left(0 . u_{1}^{\prime}(0)\right)$ and $u_{2}\left(0 . u_{2}^{\prime}(0)\right)$ and abstract boundary data $\xi_{c, s}$ of the inner structure, with regard of vanishing of the sum of corresponding boundary forms with $u=u^{3}, v=v^{3}$ :

$$
\begin{aligned}
-\frac{\hbar^{2}}{2 m_{1}} & {\left.\left[\frac{d u^{1}}{d x} \bar{v}^{1}-u^{1} \frac{d \bar{v}^{1}}{d x}\right]\right|_{x=-0}+\left.\frac{\hbar^{2}}{2 m_{1}}\left[\frac{d u^{2}}{d x} \bar{v}^{2}-u^{2} \frac{d \bar{v}^{1}}{d x}\right]\right|_{x=+0}+} \\
& +\xi_{c}^{u} \bar{\xi}_{s}^{v}-\xi_{s}^{u} \bar{\xi}_{c}^{v}=J^{1}\left(u^{1}, v^{1}\right)+J^{2}\left(u^{2}, v^{2}\right)+J^{3}(u, v) .
\end{aligned}
$$

see Appendix 1 and [12]. In particular, the sum vanishes while Datta-Das Sarma boundary conditions are imposed at the contact $x=0$ between the deposit and vacuum ( on the barrier). Those boundary conditions are defined, similarly to Datta-Das Sarma, [9], by the vector parameter $\vec{\beta}=\left(\beta_{1}, \beta_{2}, \beta_{3}\right)$ as:

$$
\frac{u^{1}}{\beta_{1}}=\frac{u^{2}}{\beta_{2}}=\frac{\xi_{-}^{u}}{\beta_{1}}, \quad \frac{\hbar^{2}}{2 m_{1}} u_{1}^{\prime} \bar{\beta}_{1}+\frac{\hbar^{2}}{2 m_{2}} u_{2}^{\prime} \bar{\beta}_{2}+\xi_{+}^{u} \bar{\beta}=0,
$$

The quantum-mechanical meaning of the similar parameter $\vec{\beta}$ in the case of the T-junction is revealed in [11]. It is defined by the spectral properties of the inner structure and the corresponding eigenfunction, [11]. Assuming that the wave-function of the electron in the carbon layer and in vacuum is a scattered wave, we represent the components of it in the deposit and in vacuum as $u^{1}=e^{i p_{1} x_{1}}+e^{-i p_{1} x_{1}} R_{1}(\lambda), u^{2}=T e^{-i p_{2} x_{2}}$. Substituting this scattering Ansatz into the above boundary conditions, we obtain an expression for the transmission coefficient $T$ 
from the deposit into vacuum $T(\lambda)=$ :

$$
\frac{\bar{\beta}_{1} \beta_{2} m_{1}^{-1 / 2}}{\left|\beta_{1}^{2}\right| m_{1}-1 / 2+\left|\beta_{2}^{2}\right| m_{2}{ }^{-1 / 2}+i\left|\beta_{3}\right|^{2}[\hbar \mathcal{M} \sqrt{2 E}]^{-1}}
$$

In the non-resonance situation, $\mathcal{M} \approx$ Const, the Datta-Das Sarma parameter $\left(1,1, e^{q a / 2}\right)$ defines the exponential small transmission rate $T \approx e^{-a q}$. Then, in the resonance situation, $\mathcal{M}=\mathcal{M}(\lambda)$ the transmission is exponentially small on the complement of the set of poles of $\mathcal{M}$, but is essentially greater at the poles $\lambda_{p} \mathcal{M}\left(\lambda_{p}\right)=\infty$, where $\mathcal{M}^{-1}=0$. In particular, for $m_{1}<<m_{2}$ we have at the poles, that

$$
T=\frac{\bar{\beta}_{1} \beta_{2} m_{1}^{-1 / 2}}{\left|\beta_{1}\right|^{2} m_{1}^{-1 / 2}+\left|\beta_{2}\right|^{2} m_{2}^{-1 / 2}} \approx \frac{\beta_{2}}{\beta_{1}},
$$

which can be essentially greater than exponential estimate $T \approx e^{-2 a v}$. Then, in the resonance situation, $\mathcal{M}=$ $\mathcal{M}(\lambda)$ based on $\beta_{3} \approx e^{v d}$ we see the peak of the transmission coefficient at the eigenvalues of the matrix $A$ which play a role of the levels of the size quantization on the barrier (with special boundary conditions on the contact of the barrier with the inner part of the deposit and the vacuum. This condition is compatible with unitarity of the full scattering matrix on the interface deposit-vacuum, if the weights $m_{1}^{-1}, m_{2}^{-1}$ are taken into account.

In our case, we have:

$$
j=-\frac{2 e v_{F}}{\hbar^{2}} \int \frac{\bar{\beta}_{1} \beta_{2} m_{1}^{-1 / 2}[f(E)-f(E+e V)] E d E}{\left|\beta_{1}\right|^{2} m_{1}^{-1 / 2}+\left|\beta_{2}\right|^{2} m_{2}^{-1 / 2}+i\left|\beta_{2}\right|^{2}[\hbar \sqrt{2 E} \mathcal{M}]^{-1}},
$$

where $\mathcal{M}=\mathcal{M}(E)$, is the Weyl-Titchmarsh function of the inner structure, see Appendix 1. just the value of $T$ at the Fermi level, with a trivial coefficient.

But in fact, the estimation of the emission current requires taking into account the density of states $\left|\frac{\partial \lambda}{\partial p}\right|^{-1}$ which requires continuity of the spectrum of surface states, while the 1D model of surface state provides only discrete resonances.

One more competing viewpoint on field emission presented, in particular, in [14] is essentially quantum, but not trivially spectral as one in [3,7]. In [14], the field emission from negative electron affinity sites on the atmosphere-GaAs interface is accompanied by optically stimulated process of oxygen adsorption. The corresponding optically induced pinning of the Fermi level leads to quenching and subsequent regeneration of the emission, which is connected in [14] with the presence of antisite defects in GaAs, formed due to the reconstruction of gallium dangling bonds on the GaAs - atmosphere interface. A possibility of the optical manipulation (monochromatic irradiation) the charges of the metastable antisite defects formed by the Ga dangling bonds is an extremely interesting aspect of the electron emission from GaAs, including enhancing the emission current due to monochromatic irradiation of the cathode under fixed exterior field. One may guess that the resonance optical excitation may generate beats of the oscillation modes which may help electrons energy to overcome the limits laid by the work function for given exterior field.

In fact, the emission current depends on the density of states $\left|\frac{d \lambda}{d p}\right|^{-1}$ - the inverse derivative of the energy with respect to the quasi-momentum, which does not arise in the $1 \mathrm{D}$ model. Fortunately, the spectrum of the size quantization in the quasi-2D periodic space charge layer is not discrete, but continuous, and consists of a sequence of spectral bands $\frac{d \lambda}{d p} \approx 0$ with a nontrivial dispersion. Attempting to explain the low threshold phenomenon, we have to develop the spectral approach for 2D periodic lattices, with regard of calculation the dispersion $\lambda\left(p^{\|}=\lambda\left(p_{1}, p_{2}\right)\right)$ on the spectral bands responsible for the transmission electrons from the spacecharge region 3 to vacuum 2, involving, together with the orthogonal to the interface $(2,3)$ component $p^{\perp}$ of the momentum, the tangential component $p^{\|}$. Planning to develop the scattering machinery for calculation of the corresponding transmission coefficient, we call the ultimate modification of the Fowler-Nordheim technique, involving the continuous spectrum with 2D quasi- momentum $p^{\|}(1+2)$, contrary to the original Fowler-Nordheim scattering approach $(1+0)$ based on scalar Schrödinger equation with 0D interface. We speculate that the continuity of spectrum arises due to the contribution of the electron flow in the carbon layer to the ultimate current. While the structure and period of the crystalline lattice in metal M may differ from the period and (hexagonal) structure of the carbon (graphene) G- layer, the problem becomes too difficult mathematically. But fortunately in our case, the metal can be considered as a bath filled with electrons, with some of natural orbitals disrupted on the metal/carbon interface. It is known, that the disrupted orbitals - the dangling bonds, see for instance [14], are restructured, transforming the interface into a quasi-2D lattice parallel to the GM interface, with a period defined by the graphene $(\mathrm{G})$-lattice, see (see Fig. 2). The calculation of resulting band-gap structure for the interacting 


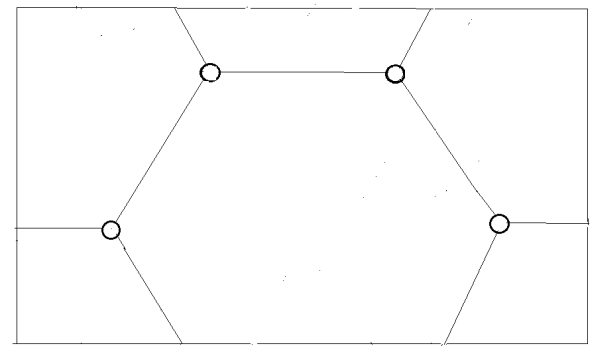

FIG. 2. Due to exterior electric field, the space-charge layer (SC) on the interface of the carbon and metal is restructured into periodic quasi-2D lattice with a rectangular period, as shown. The horizontal periodicity of the structure is not affected by the exterior electric field, normal to the interface, but the total band-gap structure is perturbed due to interaction of two periodic lattices, with equal periods, on the interface: one in metal $\mathcal{M}$, another in carbon, $\mathcal{G}$, resulting in the arisal of flat thin spectral bands of the size quantization

quasi-2D $\mathrm{M}$ and $\mathrm{G}$ lattices requires special analytical techniques using the Dirichlet-to-Neumann map, which is presented in section 3 below.

\section{Interpretation of recent experimental findings for saturation of the field emission}

Contrary to the basic $(2+1)$ model of the cathode, see Fig. 2. DN formalism, see below, section 4, allows us to calculate the dispersion function of the spectral band of the size quantization in terms of the structure of the space-charge region and the electric field applied.

But in fact the estimation of the emission current requires taking into account the density of states $\left|\frac{\partial \lambda}{\partial p}\right|^{-1}$, which can't be introduced properly in the 1D model. Fortunately, the spectrum of the size quantization in the quasi 2D periodic space charge layer is continuous, and consists of a sequence of flat spectral bands, with nontrivial dispersion $\lambda=\lambda(p)$. The ultimate version of explanation of the low threshold phenomenon, we suggest in this paper, takes into account both size quantization of electrons in the space-charge layer cv due to perturbation of the periodic structure in the space-charge layer in $1 \mathrm{D}$ vertical direction $p^{\perp}$ and the continuity of the corresponding spectrum due to periodicity of the quasi $2 \mathrm{D}$ structure in horizontal direction $p^{\|}=\left(p_{1}, p_{2}\right)$. We call the ultimate modification the " $2+1$ " model of the cathode, see Fig. 1.

DN formalism, see below, section 4, allows calculation of the dispersion function of the spectral band of the size quantization in terms of the structure of the space-charge period and electric field applied. We are able to calculate the the gradient of the dispersion function $d E=\left|\nabla_{p} E(\vec{p})\right| d p_{\perp}$ with $E=p^{2} / 2 m$. Then, for the 2-D system, the density of states in the 2-D is calculated as an integral on the surface $S$ of the cathode [15]:

$$
\rho(E)=\frac{1}{(2 \pi \hbar)^{2}} \int \frac{d S}{\left|\nabla_{p} E\right|}=\frac{1}{2 \pi \hbar^{2}} \frac{p}{\frac{\partial E}{\partial p}}=\frac{m}{2 \pi \hbar^{2}} .
$$

Recent experiments done by our group confirm the continuity of spectrum 2D-size quantization and allow us to estimate the effective mass $m^{*}$ and the de Broglie wavelength in space-charge region depending on electron's concentration $n_{e x}=\frac{1}{q} \int_{0}^{\tau} J(t) d t$, where $j=I / S, I=80 \mathrm{~A}$ is the current and $S \approx 0.75 \mathrm{~cm}^{2}-$ the area of the cathode, $\tau \approx 2 \times 10^{-9} \mathrm{~s}$. Monitoring of the current density allows us to estimate the experimental density of charges $Q=2.4 \times 10^{-7} \mathrm{Coul} / \mathrm{cm}^{2}$, which corresponds to density of electrons $n \approx 1.3 \times 10^{12} \mathrm{~cm}^{-2}$.

On another hand, from the size-quantization theory [15] the 2D density of electrons is estimated as:

$$
n_{2 D}=\frac{m^{*} k T}{2 \pi \hbar^{2}} \ln \left(1+\exp \frac{E_{0}-E_{F}}{k T}\right)
$$

Here, $m^{*}$ is the effective electron mass, $E_{0}$-the size-quantization level, $E_{F}$-the Fermi level. In our case

$$
E_{0} \approx E_{F}, \quad n_{\exp }=n_{2 D}=\frac{m^{*} k T}{2 \pi \hbar^{2}} \ln 2
$$

The de Broglie electron wavelength [15] in graphene flakes is:

$$
\lambda=\frac{2 \pi \hbar}{\sqrt{2 m^{*}(k T)}} .
$$



electron

Based on the preceding Equations (6) and (7), we estimate the electron effective mass and the de Broglie

$$
m^{*}=\frac{(2 \pi \hbar)^{2}}{2(k T)}
$$

At room temperature, we have $\lambda \approx 18 \mathrm{~nm}, m^{*} \leq 10^{-2} m_{0}$.

The potential on the space-charge region of the cathode, covered with carbon flakes is defined by the electric double layers formed by the electric field applied on the metal-carbon and carbon-vacuum interface and oriented by the outer normal looking toward vacuum. The horizontal periodicity of the cover is not affected by the field, but the vertical periodicity is perturbed by the potential well defined by the electric field, see Fig. 2. We assume that the depth of penetration of the field into the carbon layer defines the width of the potential well and the steepness of its wall. The hexagonal lattice can be represented as a periodic lattice with rectangular periods, 4 carbon atoms in each minimal period.

\section{Resonance scattering on a low-dimensional superlattice and field emission from the metal-carbon interface}

The above criticism of the resonance $0+1$ scenario of the low-threshold field emission may be resolved based on a combination of Bagraev's findings in [14] on the periodic reconstruction of dangling bonds and recent results of our experimentalists [16], revealing the role of the continuous spectrum of the size quantization. Hereafter, we construct a solvable $(2+1)$ model of a periodic 2D lattice on an interface of the Luttinger bath in metal and vacuum and calculate the scattering amplitude depending on resonance properties of the lattice period. The model can be used not only for analysis of the low-threshold emission, but also for studying the properties and dynamics stability of electric contact as a detail of a nano-device, or, generally, a quantum network. While simplest zero-range models of 2D lattices in 3D have been thoroughly investigated neglecting the "inner structure" see [17], the use of similar models with inner structure allows to interpret various instabilities of the contacts based on resonance properties of the of the inner structure.

Consider a flat 2D periodic lattice situated on the plane $z=0$. Periods $\Omega_{\vec{l}}=\left\{a_{l^{1}}<x^{1}<a_{l^{1}}+a\right\} \times$ $\left\{a_{l^{2}}<x^{2}<a_{l^{2}}+a\right\}$ in model problem [17] contain a singular points in the corners. The lattice of singular points is invariant with respect to the shifts by $a \vec{l}$. According to [17], the Laplacian restricted onto the domain $D_{0}$ of smooth functions vanishing near singular points $\left\{a_{\vec{l}}\right\}$ can be extended to Laplacian with singular zero-range potential defined by a boundary condition at the singular points imposed onto elements from the union of the domain $D_{0}$ of the restricted operator $A_{0}$ and deficiency elements selected as Green functions $G\left(x, a_{l}, \lambda_{0}\right), \Im \lambda_{0} \neq 0$ of the Laplacian. Disregarding the inner structure, one can find the scattered waves and the waveguide eigenfunction based on the Ansatzes:

$$
\begin{gathered}
\Psi^{S}(x, \nu, \lambda)=e^{i p\langle p, x\rangle}+\sum_{a_{\vec{l}}} G\left(x, a_{\vec{l}}, \lambda\right) A_{\vec{l}}^{S}, \\
\Psi^{W}(x, \nu, \lambda)=\sum_{a_{\vec{l}}} G\left(x, a_{\vec{l}}, \lambda\right) A_{\vec{l}}^{W} .
\end{gathered}
$$

The above ansatzes possess the asymptotics at the singular points:

$$
\begin{gathered}
\Psi^{S}(x, \nu, \lambda)=u^{S}=\frac{A_{\vec{l}}^{S}}{4 \pi\left|x-a_{\vec{l}}\right|}+B_{\vec{l}}^{S}+o(1) . \\
\Psi^{W}(x, \nu, \lambda)=u^{W}=\frac{A_{\vec{l}}^{W}}{4 \pi\left|x-a_{\vec{l}}\right|}+B_{\vec{l}}^{W}+o(1) .
\end{gathered}
$$

They define the boundary forms on the domain $D_{0}^{+}$of the adjoint operator $-\Delta_{0}^{+}$:

$$
J(u, v)=\left\langle-\Delta_{0}^{+} u, v\right\rangle+\left\langle u, \Delta_{0}^{+} v\right\rangle=\sum_{\vec{l}}\left[\left\langle B_{\vec{l}}(u), A_{\vec{l}}(v)\right\rangle-\left\langle A_{\vec{l}}(u), B_{\vec{l}}(v)\right\rangle\right],
$$

which vanish under "local" boundary condition, see [17] (with $\alpha=-4 \pi \mathcal{G}$ ):

$$
B_{\vec{l}}+\mathcal{G} A_{\vec{l}}=0 \text {. }
$$

with an Hermitian (real) parameter $\mathcal{G}$. The eigenfunctions $(9,10)$ are found in [17] from equations obtained via substitution of the coefficients $A_{\vec{l}}^{S}=f(\nu, \lambda) e^{i p\left\langle\nu, a_{\vec{L}}\right\rangle}, A_{\vec{l}}^{W}=e^{i\left\langle q, a_{\vec{l}}\right\rangle}$. The quasi-momentum $q(\lambda)$ for flat square 
lattice $a_{\vec{L}}$, see [17]:

$$
\mathcal{G}+\frac{i \sqrt{\lambda}}{4 \pi}+\sum_{\vec{L} \neq 0} \frac{e^{i \sqrt{\lambda}|\vec{L} a|}}{4 \pi|\vec{L} a|} e^{i\langle q, a \vec{l}\rangle}=0
$$

Similarly, the scattering amplitude is found from the Ansatz ( 9 substituted to the above boundary condition:

$$
\Psi^{S}(x, \nu, \lambda)=e^{i p\langle p, x\rangle}+f(\nu, \lambda) \sum_{a_{\vec{l}}} G\left(x, a_{\vec{l}}, \lambda\right) e^{i \sqrt{\lambda}\left\langle\nu, a_{\vec{l}}\right\rangle}
$$

which implies an equation for the amplitude $f$ :

$$
f=-\left[\mathcal{G}+\frac{i \sqrt{\lambda}}{4 \pi}+\sum_{\vec{L} \neq 0} G\left(x, a_{\vec{l}}, \lambda\right) e^{i \sqrt{\lambda}\langle\nu, a \vec{L}\rangle}\right]^{-1}
$$

In [17], the ultimate formulae are simplified, for flat rectangular lattices, based on the Poisson identity $\sum_{n \in Z} e^{i \tau n}=$ $2 \pi \sum_{n \in Z} \delta(\tau+2 \pi n)$ and explicit calculations of the lattice sums are involved. Notice that the resonance features of the amplitude at the waveguide spectral bands arise when $\sqrt{\lambda}\langle\nu, a \vec{L}\rangle=\langle q, a \vec{L}\rangle$. For the square flat lattices with no inner structure, the resonance properties and effective masses on the waveguide spectral band are defined only by the geometry of the lattice, see [17] .

In this paper, we consider flat periodic square lattice situated on the plane $z=0$, with inner structure. The presence of the inner structure defines the resonance properties of the lattice and may help to interpret instabilities of the scattering amplitude depending of properties of atoms filling the period. Consider countable set of equivalent finite-dimensional spaces $K_{\vec{l}} \equiv K$ and a virtual lattice $\oplus \sum_{\vec{L}} A_{\vec{L}}$ of equivalent operators $A_{\vec{L}} \equiv A$. We use the virtual lattice, based on operator extension procedure, to emulate the inner structure of the set of periods $\Omega_{\vec{L}}$. Selecting non-overlapping deficiency subspaces $\frac{A+i I}{A-i I} N_{i} \equiv N_{-i}$, we introduce in the defect $N \equiv N_{i}+N_{-i}$ a basis $\left\{W_{c}^{r}, W_{s}^{r}\right\}_{r}$, see Appendix 1, and calculate the boundary form of the (formal) adjoint operator $A_{0}^{+}$in terms of the decomposition coefficients $u, v \in D\left(A_{0}^{+}\right)$:

$$
u=u_{0}+\sum_{r} W_{c}^{r} \xi_{c}^{r}+W_{s}^{r} \xi_{s}^{r}=u_{0}+\sum_{r} \frac{A}{A-i I} \Xi_{c}^{r}-\frac{I}{A-i I} \Xi_{s}^{r},
$$

with $\Xi_{c}^{r}, \Xi_{s}^{r} \in N_{i}, u_{0} \in D\left(A_{0}\right)$ and the boundary form for $u=u_{0}+\frac{A}{A-i I} \Xi_{c}^{u}-\frac{I}{A-i I} \Xi_{s}^{u}, v=v_{0}+\frac{A}{A-i I} \Xi_{c}^{v-}$ $\frac{I}{A-i I} \Xi_{s}^{v}$

$$
J^{A}(u, v)=\left\langle A_{0}^{+} u, v\right\rangle-\left\langle u, A_{0}^{+} v\right\rangle=\left\langle\Xi_{c}^{u}, \Xi_{s}^{v}\right\rangle-\left\langle\Xi_{s}^{u}(u), \Xi_{c}^{v}\right\rangle
$$

The components of the solution $u=u_{0}+\frac{A}{A-i I} \Xi_{c}^{u}-\frac{I}{A-i I} \Xi_{s}^{u}$ of the homogeneous equation $A_{0}^{+} u-\lambda u=0$ are connected by the Weyl-Titchmarsh function $\mathcal{M}=P_{N_{i}} \frac{I+\lambda A}{A-\lambda I} P_{N_{i}}$ :

$$
P_{N_{i}} \frac{I+\lambda A}{A-\lambda I} P_{N_{i}} \Xi_{c}^{u}+\Xi_{s}^{u}=0 .
$$

Self-adjoint extensions of the restricted operator $A_{0}$ are parametrized by Hermitian matrices $\mathcal{G}: N_{i} \rightarrow N_{i}$, which define the domain of the corresponding extensions as restriction of $D\left(A_{0}^{+}\right)$onto the Lagrangian plane:

$$
\left.\mathcal{L}^{\mathcal{B}}=\left\{u_{0}+\left[\frac{A}{A-i I} \Xi_{c}^{u}-\frac{I}{A-i I} \Xi_{s}^{u}\right)\right]\right\}, \text { where } \Xi_{s}(u)+\mathcal{B} \Xi_{c}(u)=0,
$$

see Appendix 1 and more details in [12]. The spectrum of the extension is defined from the dispersion equation:

$$
[\mathcal{M}-\mathcal{B}] \Xi_{c}^{u}=0 \text {. }
$$

Attaching the quantum dots $A_{\vec{l}}$ to the periods $\Omega_{\vec{l}}$ of the lattice $\left\{a_{\vec{l}}\right\}$ we supply the lattice in $L_{2}\left(R_{3}\right)$ with an inner structure so that we could consider the corresponding resonance scattering problem in $L_{2}\left(R_{3}\right) \oplus \sum_{v e c L} K_{\vec{L}}$, with regard of the waveguide branch of spectrum, associated with the inner structure. 


\section{Local spectral analysis of multidimensional periodic lattices: dispersion via DN-map}

The Bloch function and dispersion of the one-dimensional periodic Schrödinger operator is found based on transfer matrix constructed of standard solutions of the Cauchy problem on the period.

$$
\begin{gathered}
-\chi^{\prime \prime}+q(x) \chi=\lambda \chi, \quad q(x+a)=q(x), \quad \chi(x+a)=\mu \chi(x), \\
\mu \equiv e^{i p a}, \quad \lambda=\lambda(p) . \\
\chi=\theta+m \varphi, \quad \theta(0)=1, \quad \theta^{\prime}(0)=0, \quad \varphi(0)=0, \quad \varphi^{\prime}(0)=1 . \\
-\theta^{\prime \prime}+q \theta=\lambda \theta, \quad-\varphi^{\prime \prime}+q \varphi=\lambda \varphi .
\end{gathered}
$$

The spectral bands $\sigma_{s}$ are defined by the condition $-1 \leq \frac{1}{2} \mathcal{T}(\lambda) \leq 1$ imposed on the $\operatorname{trace} \operatorname{Tr} \mathcal{T}(\lambda)=$ $\theta(a)+\varphi^{\prime}(a)$ of the transfer matrix:

$$
\mathcal{T}=\left(\begin{array}{cc}
\theta(a) & \varphi(a) \\
\theta^{\prime}(a) & \varphi^{\prime}(a)
\end{array}\right): \quad \mathcal{T}\left(\begin{array}{c}
\chi(0) \\
\chi^{\prime}(0)
\end{array}\right)=\left(\begin{array}{c}
\chi(a) \\
\chi^{\prime}(a)
\end{array}\right)=\mu\left(\begin{array}{c}
\chi(0) \\
\chi^{\prime}(0)
\end{array}\right) .
$$

The dispersion $\lambda=\lambda(p)$ and the positions of the spectral bands $\sigma:|\mu|=1$ are defined $-2 \leq \mu+\mu^{-1}=\operatorname{Tr} \mathcal{T}<2$, see Fig. 3.

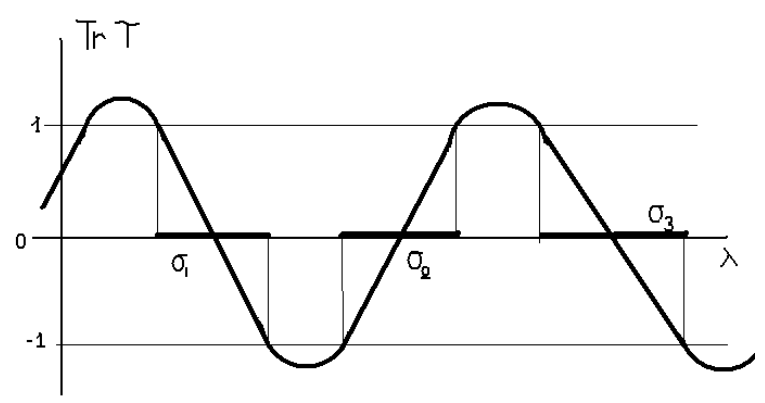

FIG. 3. The spectral bands $\sigma_{s}$ of the $1 \mathrm{D}$ periodic problem are found from the condition $-1 \leq$

$\operatorname{Tr} \mathcal{T} / 2 \leq 1$.

One can also obtain Bloch solutions from analysis of a boundary problem, by considering, instead of the standard solutions $\theta, \varphi$ of the Cauchy problem, another pair of solutions $\psi_{0}, \psi_{a}$ of the same Schrödinger equation $-\psi^{\prime \prime}+q \psi=\lambda \psi$, with the boundary data $\psi_{0}(0)=1, \psi_{0}(a)=0$ and, respectively $\psi_{a}(0)=0, \psi_{a}(a)=1$. These solutions $\psi_{0}, \psi_{a}$ of the Schrödinger equation are linearly independent if $\lambda$ is not an eigenvalue of the corresponding Dirichlet problem on the period (see Fig. 4):

$$
\left.W\left(\psi_{0}, \psi_{a}\right)\right|_{0}=-\psi_{a}{ }^{\prime}(0)=\left.W\left(\psi_{0}, \psi_{a}\right)\right|_{a}=\psi_{0}{ }^{\prime}(a)=\left.W\left(\psi_{0}, \psi_{a}\right)\right|_{a} .
$$

Then the Bloch solution can be found as a linear combination of $\psi_{0}, \psi_{a}$ in the form:

$$
\chi(x)=\chi(0) \psi_{0}(x)+\chi(a) \psi_{a}(x)=\chi(0)\left[\psi_{0}(x)+e^{i p a} \psi_{a}(x)\right]
$$

which implies:

$$
\chi^{\prime}(a)=\chi(0)\left[\psi_{0}^{\prime}(a)+e^{i p a} \psi_{a}^{\prime}(a)\right]=e^{i p a} \chi(0)\left[\psi_{0}^{\prime}(0)+e^{i p a} \psi_{a}^{\prime}(0)\right] .
$$

The quasi-momentum exponential $e^{i p a}=\mu$ is found from the quadratic equation:

$$
\left[\psi_{0}^{\prime}(a)+\mu \psi_{a}^{\prime}(a)\right]=\mu\left[\psi_{0}^{\prime}(0)+\mu \psi_{a}^{\prime}(0)\right]
$$

which can be re-written as:

$$
\mu^{2}+\frac{\psi_{0}^{\prime}(0)-\psi_{a}^{\prime}(a)}{\psi_{a}^{\prime}(0)} \mu-\frac{\psi_{0}^{\prime}(a)}{\psi_{a}^{\prime}(0)}=0
$$

Here, the coefficient in front of $-\mu$ is equal again to the trace $\operatorname{Tr} \mathcal{T}$ of the transfer-matrix:

In the multidimensional case the roles of the basic solutions $\psi_{0}, \psi_{a}$ of the boundary problems for the Schrödinger equation on the square 2D period are played by solutions associated with the boundary data forming an orthogonal basis $\left\{\psi_{s}^{\Gamma}\right\} \in L_{2}(\Gamma)$ on the boundary of the period $\Omega: \partial \Omega=\Gamma$ :

$$
-\triangle \psi_{s}+q \psi_{s}=\lambda \psi_{s},\left.\psi_{s}\right|_{\Gamma}=\psi_{s}^{\Gamma},\left\langle\psi_{s}^{\Gamma}, \psi_{t}^{\Gamma}\right\rangle_{L_{2}(\Gamma)}=\delta_{s t} .
$$



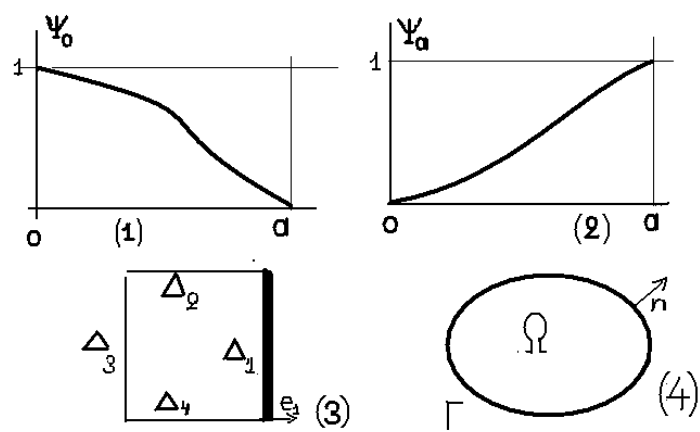

FIG. 4. Standard solutions $\psi_{0}$ (1) of the 1D boundary problem. Standard solutions $\psi_{\Delta^{1}}$ of the $2 \mathrm{D}$ boundary problem on the square.

Due to the uniqueness theorem for these elliptic equations the solutions $\left\{\psi_{s}\right\}$ are linearly independent, and their linear combinations approximate a solution of any boundary problem with the boundary data $u_{\Gamma}$ decomposed on the boundary basis.

$$
\mathcal{D N}:\left.u_{\Gamma} \longrightarrow \frac{\partial u}{\partial n}\right|_{\Gamma}
$$

Then, the Green's formula allows us to transform the matrix element into the bilinear form of the Schrödinger operator:

$$
\left\langle u_{l}, \mathcal{D} \mathcal{N} u_{m}\right\rangle=\int_{\Omega}\left[\nabla \bar{u}_{l} \nabla u_{m}+q \bar{u}_{l} u_{m}-\lambda \bar{u}_{l} u_{m}\right] d \Omega .
$$

Beginning from the solution of a sequence of Neumann problems for a smooth orthogonal basis $\left\{\rho_{s}\right\}$ in $L_{2}(\Gamma)$ :

$$
-\triangle v_{s}+q v_{s}=\lambda v_{s},\left.\frac{\partial v_{s}}{\partial n}\right|_{\Gamma}=\rho_{s}
$$

we obtain the following expression for the matrix elements of the Neumann-to-Dirichlet map:

$$
\begin{gathered}
\mathcal{N D}:\left.\left.\frac{\partial u}{\partial n}\right|_{\Gamma} \longrightarrow v\right|_{\Gamma}, \\
\left\langle\mathcal{N} \mathcal{D} \rho_{l}, \rho_{m}\right\rangle=\int_{\Omega}\left[\nabla \bar{v}_{l} \nabla v_{m}+q \bar{v}_{l} v_{m}-\lambda \bar{v}_{l} v_{m}\right] d \Omega .
\end{gathered}
$$

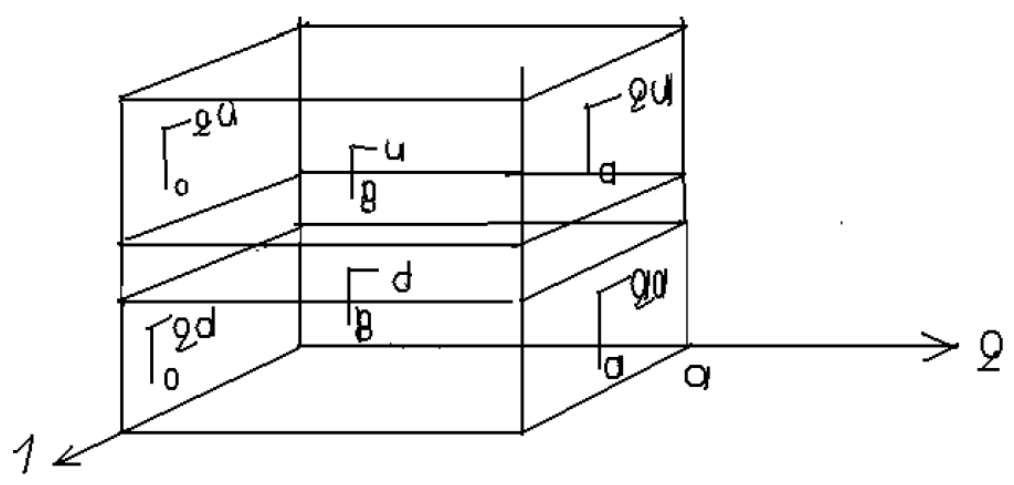

FIG. 5. Two-storied period of the periodic quasi-2D sandwich lattice

Consider the quasi-2D periodic lattice with a cubic period and the Schrödinger operator:

$$
L u=-\triangle u+q(x) u,
$$

on the lattice, with periodic potential $q\left(x^{1}, x^{2}\right)=q\left(x^{1}+m a, x^{2}+n a\right), m, n= \pm 1, \pm 2, \ldots$, zero boundary conditions on the lower and the upper lids $\Gamma_{0}^{3}: x^{3}=0, \Gamma_{h}^{3}: x^{3}=h$ of the lattice (see Fig. 5). 
In this way, the whole spectral problem on the lattice is reduced to the spectral problem on the period, with the same boundary conditions on the lids $\Gamma_{0, h}^{3}$, and the quasi-periodic conditions on the vertical walls $\Gamma_{0, a}^{1,2}$. The positive normal on $\Gamma_{a}^{1,2}$ is defined by $e_{1}, e_{2}$, and the positive normals on the walls $\Gamma_{0}^{1,2}$ are $-e_{1},-e_{2}$. The quasi-periodic boundary conditions permit us to eliminate the boundary data $\left.u\right|_{\Gamma_{0}^{1,2}},\left.\frac{\partial u}{\partial n}\right|_{\Gamma_{0}^{1,2}}$ on the walls $\Gamma_{0}^{1,2}$ :

$$
\left.u\right|_{\Gamma_{0}^{1,2}}=\left.e^{-i p_{1,2} a} u\right|_{\Gamma_{a}^{1,2}},\left.\quad \frac{\partial u}{\partial n}\right|_{\Gamma_{0}^{1,2}}=-\left.e^{-i p_{1,2} a} \frac{\partial u}{\partial n}\right|_{\Gamma_{a}^{1,2}} .
$$

Then, the quasi-periodic boundary conditions on the walls $\Gamma_{0, a}^{1,2}$ are reduced to a linear system with respect to the "independent variables" $\vec{u}=\left(u_{a}^{1}, u_{a}^{2} ;\left.\frac{\partial u}{\partial n}\right|_{\Gamma_{a}^{1}},\left.\frac{\partial u}{\partial n}\right|_{\Gamma_{a}^{2}}\right)$, with a matrix composed of the components of the $\mathcal{D N}$ on the walls:

$$
\begin{gathered}
\left(\begin{array}{c}
\left.\left.\frac{\partial u}{\partial n}\right|_{\Gamma_{0}^{1}} \frac{\partial u}{\partial n}\right|_{\Gamma_{0}^{2}} \\
-\left.e^{-i p_{1,2} a} \frac{\partial u}{\partial n}\right|_{\Gamma_{a}^{1}} \\
-\left.e^{-i p_{1,2} a} \frac{\partial u}{\partial n}\right|_{\Gamma_{a}^{2}} ^{2}
\end{array}\right)=\left(\begin{array}{c}
\frac{\partial \vec{u}_{a}}{\partial n} \\
-\mu^{-1} \frac{\partial \vec{u}_{a}}{\partial n}
\end{array}\right)=\mathcal{D N}\left(\begin{array}{c}
\left.u\right|_{\Gamma_{a}^{1}} \\
\left.u\right|_{\Gamma_{a}^{2}} \\
\left.e^{-i p_{1} a} u\right|_{\Gamma_{a}^{1}} \\
\left.e^{-i p_{2} a} u\right|_{\Gamma_{a}^{2}} ^{2}
\end{array}\right) \\
\mathcal{D N N}\left(\begin{array}{c}
\left.u\right|_{\Gamma_{a}^{1}} ^{u} \\
\left.e_{\Gamma_{a}^{2}}^{-i p_{1} a} u\right|_{\Gamma_{a}^{1}} \\
\left.e^{-i p_{2} a} u\right|_{\Gamma_{a}^{2}}
\end{array}\right) \equiv \mathcal{D N}\left(\begin{array}{c}
\vec{u}_{a} \\
\mu^{-1} \vec{u}_{a}
\end{array}\right)
\end{gathered}
$$

Here, $\mu=\left[\mu_{1}, \mu_{2}\right]=\left[e^{i p_{i} a}, e^{1 p_{2} a}\right]$ is a diagonal matrix. The DN-map $\mathcal{D N}$ can be represented in matrix form with $2 \times 2$ blocks $\mathcal{D} \mathcal{N}_{\alpha \beta}^{i k}$ connecting the Dirichlet data on $\Gamma_{\beta}^{k}$ to the Neumann data on $\Gamma_{\alpha}^{i}$.

Matrix elements of the DN map connect the Dirichlet data on $\Gamma_{\alpha}^{i k}$ with Neumann data on $\Gamma_{\alpha^{\prime}}^{j l}, \alpha, \alpha^{\prime}=o, a$ :

$$
\begin{aligned}
& \left(\begin{array}{ll}
\mathcal{D} \mathcal{N}_{a a}^{11} & \mathcal{D N}_{a a}^{12} \\
\mathcal{D} \mathcal{N}_{a a}^{21} & \mathcal{D N}_{a a}^{22}
\end{array}\right) \equiv \mathcal{D} \mathcal{N}_{a a},\left(\begin{array}{ll}
\mathcal{D N}_{a 0}^{11} & \mathcal{D N}_{a 0}^{12} \\
\mathcal{D N}_{a 0}^{21} & \mathcal{D N}_{a 0}^{22}
\end{array}\right) \equiv \mathcal{D N}_{a 0} \\
& \left(\begin{array}{ll}
\mathcal{D} \mathcal{N}_{0 a}^{11} & \mathcal{D} \mathcal{N}_{0 a}^{12} \\
\mathcal{D} \mathcal{N}_{0 a}^{21} & \mathcal{D} \mathcal{N}_{0 a}^{22}
\end{array}\right) \equiv \mathcal{D} \mathcal{N}_{0 a},\left(\begin{array}{cc}
\mathcal{D N}_{00}^{11} & \mathcal{D N}_{00}^{12} \\
\mathcal{D} \mathcal{N}_{00}^{21} & \mathcal{D N}_{00}^{22}
\end{array}\right) \equiv \mathcal{D} \mathcal{N}_{00}
\end{aligned}
$$

Then the DN-map is represented by the block-matrix:

$$
\mathcal{D N}=\left(\begin{array}{ll}
\mathcal{D N}_{a a} & \mathcal{D N}_{a 0} \\
\mathcal{D} \mathcal{N}_{0 a} & \mathcal{D} \mathcal{N}_{00}
\end{array}\right)
$$

with blocks mapping the data $\vec{u}_{a}, \vec{u}_{0}$ onto the positive normal derivatives $\frac{\partial \vec{u}_{a}}{\partial n}, \frac{\partial \vec{u}_{0}}{\partial n}$.

In particular, the 0 -components of the Bloch function can be eliminated based on $\vec{u}_{0}=\mu^{-1} \vec{u}_{a}$, $\frac{\partial \vec{u}_{0}}{\partial n}=$ $-\mu^{-1} \frac{\partial \vec{u}_{a}}{\partial n}$, which implies the following linear homogeneous system for the data $\left(\vec{u}_{a}, \frac{\partial \vec{u}_{a}}{\partial n}\right)$ of the Bloch-function:

$$
\left(\begin{array}{c}
\frac{\partial \vec{u}_{a}}{\partial n} \\
-\mu^{-1} \frac{\partial \vec{u}_{a}}{\partial n}
\end{array}\right)=\left(\begin{array}{cc}
\mathcal{D N}_{a a} & \mathcal{D N}_{a 0} \\
\mathcal{D} \mathcal{N}_{0 a} & \mathcal{D N}_{00}
\end{array}\right)\left(\begin{array}{c}
\vec{u}_{a} \\
\mu^{-1} \vec{u}_{a}
\end{array}\right)
$$


Eliminating $\frac{\partial \vec{u}_{a}}{\partial n}$ we conclude that a nontrivial solution of the equation (30) exists if and only if zero is an eigenvalue of the operator:

$$
\left[\mu \mathcal{D N}_{00} \mu^{-1}+\mu \mathcal{D} \mathcal{N}_{0 a}+\mathcal{D} \mathcal{N}_{a a}+\mathcal{D} \mathcal{N}_{a 0} \mu^{-1}\right] \vec{u}_{a}=0
$$

Then, the Bloch function is obtained as a solution of the boundary problem for the Schrödinger equation:

$$
-\triangle \chi+q \chi=\lambda \chi,\left.\quad \chi\right|_{\Delta_{a}^{1,2}}=u_{a}^{1,2},\left.\quad \chi\right|_{\Delta_{0}^{1,2}}=e^{-i p_{1,2} a} u_{a}^{1,2} .
$$

Equation (31) is an analog of the quadratic equation (23), however questions on the existence of the corresponding solution of it in the general case is not trivial, because we can't use the classical determinant condition of existence of non-trivial solutions of the homogeneous equation (31).

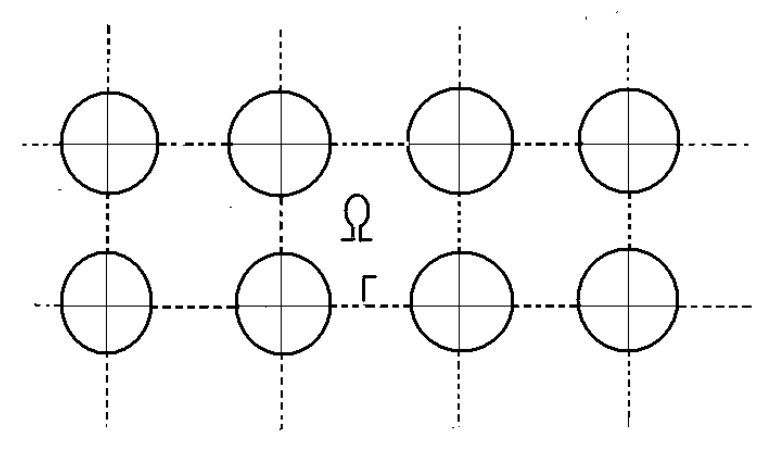

FIG. 6. 2D periodic lattice with romboidal periods

\section{Examples of iso-energetic surfaces associated with solvable models of periodic lattices}

Consider a typical example of a 2D lattice generated by a non-dimensional Schrödinger operator with real periodic potential obtained via restriction of Yukawa potential on the romboidal period (see Fig. 6) framed by the arcs of circles radius 0.05 centered on the corners of the square $1.1 \times 1.1$ and by the central intervals $\Gamma_{\alpha}^{i}$ length $\delta$ on the sides of the period. We choose the contacts $\Gamma_{\alpha}^{i}$ in form of intervals $0<\gamma<\delta^{i}$ centered at the mid-points $0_{\alpha}^{i}$ of the corresponding sides of the square period and span the contact spaces by $\sqrt{2 / \delta^{i}} \sin l \pi \gamma / \delta^{i}$ and use the basic equation (31). The direction of vector $\nu$ is defined by the angle $\varphi=0,15^{0}, 30^{0}, 45^{0}$ between the orth $e_{1}$ and $\nu$.

For strong Yukawa potential the dispersion function $\lambda(|p|)$ with $3 \mathrm{D}$ contact spaces $l=1,2,3$ on the on the contacts is calculated for selected angles and is represented based on straightforward computing for the corresponding DN-map.

Our numerical experiments showed that beginning from $\operatorname{dim} N=3$ the shape of the dispersion function in the domain of low energy reveals clear features of stability, which gives a good reason to assume that the finite dimension of the contact subspace already allows to construct a realistic soluble model of the Schrödinger operator with Yukawa potential on the above square lattice (see Fig. 7, Fig. 8, Fig. 9).

Interesting resonance properties are revealed by Heine-Abarenkov potential constructed on a period as a potential well surrounded by the thick wall. The Dirichlet problem on the "romboidal" period for the corresponding Schrödinger operator has a single simple eigenvalue represented by an isolated pole of the DN-map plus a regular correcting term.

The rational approximation of the corresponding DN-map bordered by the projections on the corresponding $1 \mathrm{D}$ contact spaces $N_{1}, N_{2}$, spanned by $\sqrt{2 / \delta^{i}} \sin l \pi \gamma / \delta^{i}, l=1$, for low temperature on the corresponding small temperature interval centered at the lowest resonance eigenvalue $\lambda_{1}$ has the form:

$$
A \frac{Q}{\lambda-\lambda_{1}}+B=A_{1} \frac{\left(\begin{array}{cc}
Q_{a a} & Q_{a 0} \\
Q_{0 a} & Q_{00}
\end{array}\right)}{\lambda-\lambda_{1}}+\left(\begin{array}{cc}
B_{a a} & B_{a 0} \\
B_{0 a} & B_{00}
\end{array}\right),
$$

with an one-dimensional orthogonal projection $Q$ and a constant Hermitian matrix. 


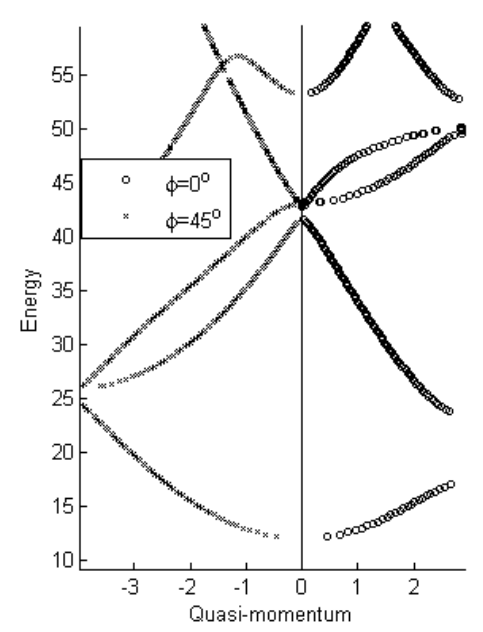

FIG. 7. Sections of the dispersion surface of the Yukawa lattice.

We select:

$$
\begin{gathered}
Q_{a a}=\frac{1}{2}\left(\begin{array}{cc}
\left.\nu_{1}\right\rangle\left\langle\nu_{1}\right. & 0 \\
0 & 0
\end{array}\right) ; \quad Q_{a 0}=\frac{1}{2}\left(\begin{array}{cc}
0 & \left.\nu_{1}\right\rangle\left\langle\nu_{2}\right. \\
0 & 0
\end{array}\right) ; \\
\left.Q_{0 a}=\frac{1}{2}\left(\begin{array}{cc}
0 & 0 \\
\left.\nu_{2}\right\rangle\left\langle\nu_{1}\right. & 0
\end{array}\right) ; \quad Q_{00}=e_{0}\right\rangle\left\langle e_{0}=\frac{1}{2}\left(\begin{array}{cc}
0 & 0 \\
0 & \left.\nu_{2}\right\rangle\left\langle\nu_{2}\right.
\end{array}\right),\right.
\end{gathered}
$$

see Fig. 8. The regular term $B$ depends on the upper eigenvalues and eigenfunctions.

We consider an example selecting the regular term as:

where:

$$
B=\left(\begin{array}{cc}
B_{a a} & B_{a 0} \\
B_{0 a} & 0
\end{array}\right),
$$

$$
\begin{gathered}
B_{00}=\left(\begin{array}{ll}
0 & 0 \\
0 & 0
\end{array}\right), B_{a a}=\left(\begin{array}{ll}
1 & 0 \\
0 & 0
\end{array}\right), \\
B_{a 0}=b_{a 0}\left(\begin{array}{cc}
0 & \left.\nu_{1}\right\rangle\left\langle\nu_{2}\right. \\
\left.\nu_{2}\right\rangle\left\langle\nu_{1}\right. & 0
\end{array}\right), B_{0 a}=b_{0 a}\left(\begin{array}{cc}
0 & \left.\nu_{2}\right\rangle\left\langle\nu_{1}\right. \\
\left.\nu_{1}\right\rangle\left\langle\nu_{2}\right. & 0
\end{array}\right) .
\end{gathered}
$$

Elimination of the variables $u_{0}^{\prime}, u_{a}^{\prime}, u_{0}$ gives an equation for the 2-vector $u_{a}$ :

$$
\begin{gathered}
\frac{A}{\lambda-\lambda_{1}^{D}}\left[\mu Q_{0 a}^{N}+\mu Q_{00}^{N} \mu^{-1}+Q_{a a}^{N}+Q_{a 0}^{N} \mu^{-1}\right] \vec{u}_{a}+ \\
{\left[\mu B_{0 a}^{N}+\mu B_{00}^{N} \mu^{-1}+B_{a a}^{N}+B_{a 0}^{N} \mu^{-1}\right] \vec{u}_{a} \equiv} \\
{\left[\frac{A \mathcal{D}_{Q}(\lambda, p)}{\lambda-\lambda_{1}^{D}}+\mathcal{D}_{B}(\lambda, p)\right] \vec{u}_{a}=0 .}
\end{gathered}
$$

The corresponding equation (34) has a nontrivial solution $\vec{u}_{a}$ if the determinant of the corresponding $2 \times 2$ matrix:

$$
A \mathcal{D}_{Q}+\left(\lambda-\lambda_{1}^{D}\right) \mathcal{D}_{B}(\lambda, p)
$$

vanishes. This condition yields the dispersion equation $\lambda=\lambda(\vec{p})$, with the quasi-momentum $\vec{p}$ defined by the quasi-momentum exponentials $\mu=\operatorname{diag}\left(\mu_{1}, \mu_{2}\right)=\operatorname{diag}\left(e^{i p_{1}}, e^{i p_{2}}\right)$.

In the case when the Heine-Abarenkov potential well is deep enough, there may be several eigenvalues with eigenfunctions localized in the well.

In particular, there is an eigenfunction symmetric with respect to reflection in the line connecting the mid points of $\Gamma_{0}^{1}, \Gamma_{a}^{1}$ and antisymmetric with respect to reflection in the line connecting the mid-points of $\Gamma_{0}^{2}, \Gamma_{a}^{2}$, or vice versa. The corresponding eigenvalue is non-degenerated if the potential is not symmetric with respect to the 


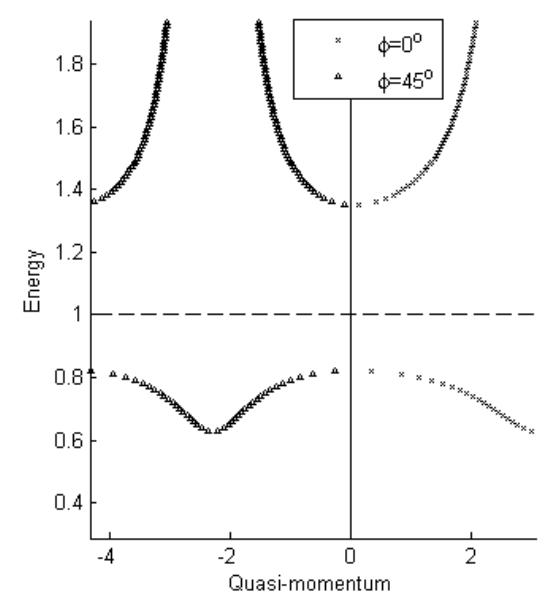

FIG. 8. Sections of the dispersion surface of an abstract model emulating Heine-Abarenkov potential with the lower resonance eigenvalue $\lambda_{1}=1$ (punctured line)

change of the variables $1 \rightarrow 2,2 \rightarrow 1$. The corresponding DN-map is characterized by the polar term $A Q$ with the projection:

$$
Q=\frac{1}{2}\left(\begin{array}{cccc}
\left.\nu_{1}\right\rangle\left\langle\nu_{1}\right. & 0 & \left.-\nu_{1}\right\rangle\left\langle\nu_{1}\right. & 0 \\
0 & 0 & 0 & \left.-\nu_{1}\right\rangle\left\langle\nu_{1}\right. \\
\left.-\nu_{1}\right\rangle\left\langle\nu_{1}\right. & 0 & 0 & 0 \\
0 & \left.-\nu_{1}\right\rangle\left\langle\nu_{1}\right. & 0 & \left.\nu_{1}\right\rangle\left\langle\nu_{1}\right.
\end{array}\right) .
$$

If the correcting term is selected as above, we obtain the dispersion curves in various direction as shown one Fig. 5:

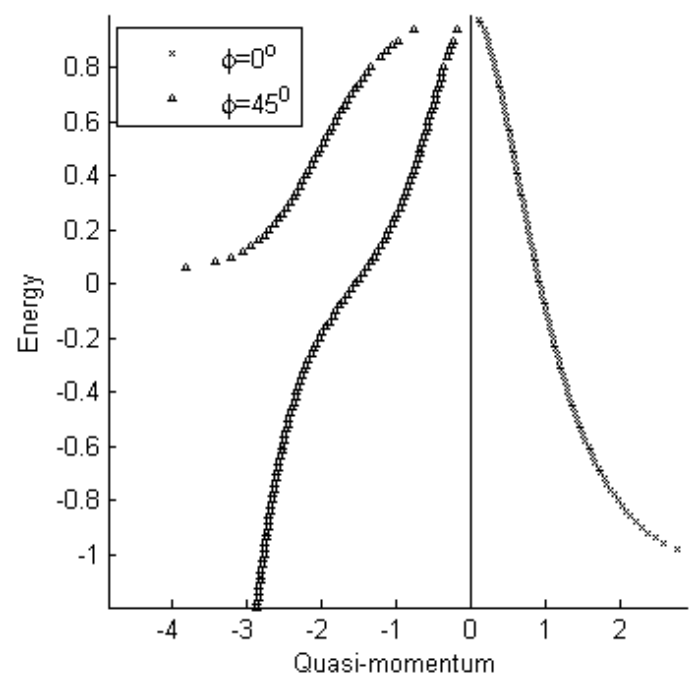

FIG. 9. Sections of the dispersion surface of the abstract model emulating Heine-Abarenkov potential with resonance eigenvalue $\lambda_{2}>\lambda_{1}$

In fact, our proposal has a softer nature ( in the sense of V. Arnold), due to the freedom of selection of the rational approximation ( probably a multi-pole- approximation) for the real material lattice or sandwich on the resonance domain:

$$
\mathcal{D N} \approx \sum_{s \leq m} A_{s} \frac{Q_{s}}{\lambda-\lambda_{s}}+P_{m}(\lambda)
$$


the choice of the contacts/contact subspaces and temperature (interval of admissible values of energy). We speculate that this problem, although it appears to be a mathematical one, lies outside of mathematics, on the interface with quantum chemistry, with its specific system of notions (covalent bonds, etc...) and methods.

\section{Appendix 1: Lagrangian version of the operator extension algorithm}

John von Neumann - nearly 90 years ago - tried to attract attention of physicists to the basic difference between symmetric and self-adjoint operators, see [18], and proposed a geometrical solution to the most important problem of construction of a self-adjoint extension of a symmetric operator. Unfortunately, this solution was not, at that time, properly recognized by physicists. A few years later, E. Fermi, attempting to describe scattering of neutrons by nuclei, considered the Laplacian on a domain of smooth $L_{2}\left(R_{3}\right)$ functions with typical singularity at the origin $u=\frac{A^{u}}{4 \pi|x|}+B^{u}+O(|x|)$ and discovered that the boundary form of the Laplacian:

$$
J(u, v)=\lim _{\delta \rightarrow 0} \int_{|x|>\delta}[-\Delta \bar{u} v+\bar{u} \Delta v] d^{3} x=\bar{A}^{u} B^{v}-\bar{B}^{u} A^{v}
$$

vanishes under the boundary condition $B=\gamma A$ with real $\gamma$. Later probably used another approach to the problem of extending of a symmetric operator to the corresponding self-adjoint, which yields a convenient formula for the scattered waves, see [19]. Though the conventional proof of self-adjointness of the Laplacian under the above boundary conditions was proposed 25 years later, see [20], the approach to operator extension based on the boundary form proved to be extremely efficient, see for instance [12,13,21] ${ }^{1}$ In 1970 's, it was modified by introducing the inner structure into zero-range potential, see $[12,13]$, that allowed consideration of resonance interaction, which allows admission of fitting based on asymptotics Dirichlet-to-Neumann map of the corresponding unperturbed problem, see [22]. This approach allows one to develop an analytic perturbation technique for embedded eigenvalues, based on two step analytic perturbation procedure - a quantum Jump-Start analog of the corresponding classical techniques developed by Poincare [23] and, in particular, to propose a convenient solvable model for Quantum Networks, supplied with inner structure on the nodes, see [24] ${ }^{2}$.

Hereafter, we produce a brief review of the Lagrangian operator extension techniques for an abstract operator, presenting the corresponding symplectic boundary form in terms of appropriate analog of boundary values, and imposing the corresponding boundary conditions, to select a domain of the relevant self-adjoint extension as a Lagrangian plane of the symplectic boundary form.

Consider a finite Hermitian matrix $A$ in a finite-dimensional Hilbert space $A: K \rightarrow K, \operatorname{dim} K=k$. Select a deficiency subspace $N_{i} \in K, \operatorname{dim} N_{i}<k / 2$, such that does not overlap with $N_{-i}=\frac{A+i I}{A-i I} N_{i}$ and consider the restriction $A_{0}=\left.A\right|_{D_{0}}$ onto the subspace $D_{0} \equiv(A-i I)^{-1}\left[K \ominus N_{i}\right]$. The restricted operator is not densely defined, but its formal adjoint can be defined by J. von Neumann formulae on the defect $N=N_{i}+N_{-i}$ as $A^{+} n_{i}+i n_{i}=0, n_{i} \in N_{i}, A^{+} n_{-i}-i n_{-i}=0, n_{-i} \in N_{-i}$, see [25], where the operator extension procedure is developed for $A_{0}$. In $[12,13]$, see also references therein, a simplectic version of the operator extension procedure was proposed for $A_{0}$, which is convenient for manufacturing zero-range perturbations with inner structure for differential operators, see for instance $[12,13,24]$. In particular, a quantum dot attached to the star-graph $\Gamma=\cup \gamma_{l}$ is modeled in [24] based on zero-range potential with inner structure while reducing $L \rightarrow L_{0}$ the Schrödinger operator by the condition of vanishing elements of the domain $D\left(L_{0}\right)$ near the knot $x=0$ of the star, such that the adjoint opeartor $L_{0}^{+}$has the boundary form represented as a sum over all branches $\gamma_{l}$ of the star graph $\Gamma$, meeting at the node $x=0$ :

$$
J^{e x t}(u, v)=\sum_{l} \int_{\gamma_{l}}\left[-\bar{u}_{l}^{\prime \prime} v_{l}+\bar{u}_{l} v_{l}^{\prime \prime}\right] d x=\left.\sum_{l}\left[-\bar{u}_{l}^{\prime} v_{l}+\bar{u}_{l} v_{l}^{\prime}\right]\right|_{0}
$$

\footnotetext{
${ }^{1}$ I. M. Gelfand attracted attention of mathematicians to importance of development a Lagrangian approach to operator extensions, based on selecting a Lagrangian plane in the domain of the adjoint operator, which would serve an alternative to the J. von Neumann geometrical construction, [26].

${ }^{2}$ Notice, that I. Prigogine in 1973 formulated the hypothesis on the validity of the Poincare two-step algorithm of analytic perturbation procedure for quantum problems, see [27], but could not prove it, because selected an incorrect anzsatz was selected for the corresponding Intermediate operator. The hypothesis was later proved for Quantum Networks based on the correct ansatz, [22], for the intermediate operator, presented in the form of zero-range model with an inner structure, constructed with use of Lagrangian technique of operator extension procedure [23]
} 
with differentiation in an outgoing direction at the node. The boundary form of the inner Hamiltonian $A$ is calculated in a special representation of the boundary form $J^{i n t}(u, v)$, constructed based on an orthogonal basis $\left\{e_{l}\right\} \subset N_{i}$ :

$$
\begin{gathered}
\left\{W_{+}^{l}\right\}=\left\{\frac{1}{2}\left[e_{l}+\frac{A+i I}{A-i I} e_{l}\right]\right\}=\left\{\frac{A}{A-i I} e_{l}\right\} \text { and }\left\{W_{-}^{l}\right\}=\left\{\frac{1}{2}\left[e_{l}-\frac{A+i I}{A-i I} e_{l}\right]\right\}=\left\{-\frac{1}{A-i I} e_{l}\right\} \\
J^{e x t}(u, v)=\sum_{l} \int_{\gamma_{l}}\left[-\bar{u}_{l}^{\prime \prime} v_{l}+\bar{u}_{l} v_{l}^{\prime \prime}\right] d x=\left.\sum_{l}\left[-\bar{u}_{l}^{\prime} v_{l}+\bar{u}_{l} v_{l}^{\prime}\right]\right|_{0}
\end{gathered}
$$

with differentiation in an outgoing direction at the node. The boundary form of the inner Hamiltonian $A$ is calculated in a special representation of the boundary form $J^{i n t}(u, v)$, constructed based on an orthogonal basis $\left\{e_{l}\right\} \subset N_{i}$ :

$$
\left\{W_{+}^{l}\right\}=\left\{\frac{1}{2}\left[e_{l}+\frac{A+i I}{A-i I} e_{l}\right]\right\}=\left\{\frac{A}{A-i I} e_{l}\right\} \text { and }\left\{W_{-}^{l}\right\}=\left\{\frac{1}{2}\left[e_{l}-\frac{A+i I}{A-i I} e_{l}\right]\right\}=\left\{-\frac{1}{A-i I} e_{l}\right\} .
$$

Then, each element from the defect $N=N_{i}+N_{-i}$ can be represented as $u=\sum_{l} \frac{A}{A-i I} \xi_{+}^{l}-\frac{1}{A-i I} \xi_{-}^{l} \equiv$ $\frac{A}{A-i I} \vec{\xi}_{+}^{u}-\frac{1}{A-i I} \vec{\xi}_{-}^{u}$, and the vectors $\vec{\xi}_{ \pm}^{u} \in N_{i}$ play roles of the boundary data. The boundary form of the formal adjoint operator $A_{0}^{+}$is calculated on elements $u, v \in N$ from the defect as:

$$
J^{i n t}(u, v)=\left\langle A^{+} u, v\right\rangle-\left\langle u, A^{+} v\right\rangle=\left\langle\vec{\xi}_{+}^{u}, \vec{\xi}_{-}^{v}\right\rangle-\left\langle\vec{\xi}_{-}^{u}, \vec{\xi}_{+}^{v}\right\rangle
$$

The ultimate formula is valid tot only on the defect, but on the whole space $K=D_{0}+N$ with $A_{0}^{+}$extended from $N$ onto $D_{0}$ as $A_{0}$, that is, for $u=u_{0}+u_{N}$ :

$$
A^{+}\left(u_{0}+u_{N}\right)=A_{0} u_{0}+A_{0}^{+} u_{N}
$$

so that the addendum $A_{0} u_{0}$ does not contribute to the boundary form $J^{e x t}(u, v)$, and $J^{e x t}(u, v)=\left\langle A^{+} u_{N}, v_{N}\right\rangle-$ $\left\langle u_{N}, A^{+} v_{N}\right\rangle$. In particular, the boundary data $\xi_{ \pm}^{u}$ for a solution of the homogeneous adjoint equation:

$$
A^{+}\left(u_{0}+u_{N}\right)-\lambda\left(u_{0}+u_{N}\right)=0
$$

are connected by the Krein function $M(\lambda)=\left.P_{N_{i}} \frac{I+\lambda A}{A-\lambda I}\right|_{N_{i}}$ as:

$$
\xi_{-}^{u}+\left.P_{N_{i}} \frac{I+\lambda A}{A-\lambda I}\right|_{N_{i}} \xi_{+}^{u}=0 .
$$

A self-adjoint extension of the restricted operator $A_{0}$ on the defect is constructed as a part of the extended adjoint operator onto the Lagrangian plane $N^{B}$ in the defect, submitted to the boundary condition:

$$
\vec{\xi}_{-}^{u}+B \vec{\xi}_{+}^{u}=0
$$

with an Hermitian operator $B: N_{i} \rightarrow N_{i}$ The boundary form (41) vanishes on the plane. Then, the corresponding self-adjoint extension of an original restricted operator $A_{0}$ is defined as a sum $A_{0}+A^{B}$ acting according to the von Neumann formulae respectively in $D_{0}$ and in $N^{B} \subset N$ on elements:

$$
u=u_{0}+\frac{A}{A-i I} \vec{\xi}_{+}^{u}-\frac{I}{A-i I} \vec{\xi}_{-}^{u}
$$

with the boundary values connected by the above boundary conditions .

The spectrum of the extension is defined by the Krein function:

$$
P_{N_{i}} \frac{I+\lambda A}{A-\lambda I} P_{N_{i}} \equiv \mathcal{M}(\lambda)
$$

which connects the boundary values $\vec{\xi}_{ \pm}^{u}$ of the solution $u$ of the homogeneous adjoint equation $A^{+} u-\lambda u=0$ :

$$
\vec{\xi}_{-}^{u}+\mathcal{M}(\lambda) \vec{\xi}_{+}^{u}=0
$$

and the spectrum of the extension $A^{B}$ is defined by the singularities of the corresponding ratio

$$
\frac{I}{B-\mathcal{M}(\lambda)},
$$


which serves the inner factor in the Krein formula for the resolvent of the extension:

$$
\frac{I}{A^{B}-\lambda I}=\frac{I}{A-\lambda I}+\frac{A+i I}{A-\lambda I} P_{N_{i}} \frac{I}{B-\mathcal{M}(\lambda)} P_{N_{i}} \frac{A-i I}{A-\lambda I} .
$$

\section{Appendix 2: Solvable models of selected one-body spectral problems}

In this section we construct a one-body model of a quantum dot $\Omega$ attached to the node of a $1 \mathrm{D}$ quantum star-graph $\Gamma=\Gamma_{1} \cup \Gamma_{2} \cup \Gamma_{3} \ldots \Gamma_{n}$ and a one-body model of 1D periodic chain. Despite selection of simplest algebraic parameters for the corresponding solvable models, they have rich spectral propeties which can be easily monitored due to the algebraic nature of the parameters selected. Both models are constructed via Lagrangian version of the operator extension procedure, with regard of the symplectic boundary forms $J$ balancing to zero on Lagrangian planes, selected based on the corresponding boundary conditions.

\section{Quantum dot attached to the node of a quantum graph}

On a star-graph $\Gamma=\Gamma_{1} \cup \Gamma_{2} \cup \Gamma_{3} \ldots \Gamma_{n}$ of straight shoulders $\Gamma_{s}$ attached to a compact domain $\Omega$ with a smooth boundary, we consider a spectral problem for a the $1 \mathrm{D}$ Schrödinger equation $L^{\Gamma}=-\vec{u}_{\Gamma}^{\prime \prime}$ in $L_{2}\left(\Gamma, E^{\Gamma}\right)$ with $E^{\Gamma}=C_{n}$, with regard of appropriate boundary condition connecting it with the Schrödinger operator $L_{\Omega} u_{\Omega}=$ $-\Delta u_{\Omega}+V u_{\Omega}$ on the domain $\Omega$ supplied with the zero boundary condition $L_{2}(\partial \Omega) \ominus E^{\Omega}$. A bond $B$ is imposed on the contact $E^{\Omega} \times E^{\Gamma}$ with the projection $P^{\Omega} \equiv P_{E^{\Omega}}$. This was done with the consideration of the boundary forms calculated on both sides of the contact $\Gamma \cap \partial \Omega$ in the corresponding contact subspaces $C_{n}=E^{\Gamma}$, $E^{\Omega}$, with $P^{\Omega} \equiv P_{E^{\Omega}}:$

$$
J_{\Gamma}\left(u_{\Gamma}, v_{\Gamma}\right) \equiv\left\langle\vec{u}_{\Gamma}^{\prime}, \vec{v}_{\Gamma}\right\rangle-\left\langle\vec{u}_{\Gamma}, \vec{v}_{\Gamma}^{\prime}\right\rangle, J_{\Omega}\left(u_{\Omega}, v_{\Omega}\right) \equiv-\left\langle\frac{\partial u_{\Omega}}{\partial n}, P^{\Omega} v_{\Omega}\right\rangle+\left\langle P^{\Omega} u_{\Omega}, \frac{\partial v_{\Omega}}{\partial n}\right\rangle
$$

and a boundary condition imposed with an Hermitian matrix:

$$
B=\left(\begin{array}{cc}
0 & b_{e i} \\
b_{i e} & a_{i i}
\end{array}\right),\left(\begin{array}{c}
\vec{u}_{\Gamma}^{\prime} \\
-P^{\Omega} \frac{\partial u_{\Omega}}{\partial n}
\end{array}\right)+B\left(\begin{array}{c}
\vec{u}_{\Gamma} \\
P^{\Omega} u_{\Omega}
\end{array}\right)=0,
$$

with an Hermitian operator $a_{i i}: E^{\Omega} \rightarrow E^{\Omega}$ and $b_{e i}: E^{\Omega} \rightarrow E^{\Gamma}, b_{i e}=b_{e i}^{+}$. We denote by $\mathcal{M} \equiv-P^{\Omega} \mathcal{D N} P^{\Omega}=$ $\sum_{l} \frac{\left.P^{\Omega} \frac{\partial \varphi_{l}}{\partial n}\right\rangle\left\langle\frac{\partial \varphi_{l}}{\partial n} P^{\Omega}\right.}{\lambda_{l}-\lambda}$ the framed DN-map ${ }^{3}$ of the quantum dot:

$$
-\Delta u_{\Omega}+V u_{\Omega}=\lambda u_{\Omega}, \mathcal{D N}:\left.u_{\Omega} \rightarrow \frac{\partial u_{\Omega}}{\partial n}\right|_{\partial \Omega},
$$

and rewrite equation (49), for the scattering Ansatz $e^{i p x} \vec{e}+e^{-i p x} S \vec{e}=\vec{u}, p^{2}=\lambda$ :

$$
\begin{array}{ccc}
i p(I-S) e+ & b_{e i} P^{\Omega} u_{\Omega}= & 0 \\
{\left[\mathcal{M}+a_{i i}\right] P^{\Omega} u_{\Omega}+} & b_{i e}(I+S) e= & 0 .
\end{array}
$$

Eliminating $P^{\Omega} u_{\Omega}$ and introducing $b_{e i} \frac{1}{\mathcal{M}+a_{i i}} b_{i e} \equiv \mathcal{N}^{b}(\lambda)$, we obtain an expression for the scattering matrix:

$$
S(p)=\frac{i p-\mathcal{N}^{b}}{i p+\mathcal{N}^{b}}, \text { with } \Im \mathcal{N}^{b} \Im \lambda>0,
$$

in terms of the framed Neumann-to-Dirichlet map $\mathcal{N}^{b}$.

Though the Dirichlet-to-Neumann map of the 3D domain $\Omega$ can be computed with standard programs, see for instance [28], yet it is also convenient to substitute it by perturbation analysis [] using finite-dimensional approximation, taking into account only a finite number of eigenvalues $\lambda_{l}$ or substituting the quantum dot by zero-range potential with inner structure, see $[12,13]$. In particular, for a 1D framed DN map, the framed ND-map $\mathcal{N}^{b} \equiv b_{e i} \frac{1}{\mathcal{M}+a_{i i}} b_{i e}$ has generally has asymptotes at infinity $\lambda \rightarrow \infty$ :

$$
\mathcal{N}^{b}(\lambda)=\hat{A} \lambda+\hat{A}_{0}+\sum_{l=1}^{k} \frac{A_{l}}{\lambda_{l}-\lambda},
$$

with $\hat{A} \geq 0, A_{0}=\bar{A}_{0}, A_{l} \geq 0$. It is possible to select a finite-dimensional self-adjoint operator $A: K \rightarrow K$ and an interaction $B$, with non-overlapping deficiency subspaces $N_{i}, N_{-i}$ such that, being attached to node of the

\footnotetext{
${ }^{3}$ The above formal series is actually divergent, but may be properly regularized, see for instance [29].
} 
above star-graph $\Gamma$, plays a role of an inner Hamiltonian of a quantum dot with the scattering matrix (51) on the star-graph, with the boundary conditions .

\section{A solvable model of a discrete lattice}

Consider a 1D periodic lattice ( a chain) of equivalent quantum dots $\Omega \equiv \Omega_{l}$ arising of equivalent finitedimensional operators $A_{l} \equiv A: K_{l} \rightarrow K_{l}$, with equivalent non-overlapping 1D deficiency subspaces $N_{i}^{l}, N_{-i}^{l}$. Each quantum dot is substituted by the zero-range potential with an inner structure as described in Appendix 1:

$$
\left.\xi_{+} \sim P_{E} u^{\Omega}\right|_{\Gamma}, \quad \xi_{-} \sim-\left.P_{E} \frac{\partial u^{\Omega}}{\partial n}\right|_{\Gamma}, \xi_{-}+\mathcal{M} \xi_{+}=0
$$

and a pair of infinite-dimensional vectors $\vec{\xi}_{+}=\sum_{l} \xi_{-}^{l}, \vec{\xi}_{-}=\sum_{l} \xi_{+}^{l}$, which play a role of the boundary values for the whole chain. We choose the simplest translation - invariant boundary conditions, connecting the nearest neighbors in the chain as:

$$
\xi_{-}^{l}+b^{+} \xi_{+}^{l-1}+b_{0}+b \xi_{+}^{l+1}=0, \text { or, alternatively } \xi_{+}^{l}+c^{-} \xi_{-}^{l-1}+c_{0}+c \xi_{-}^{l+1}=0 .
$$

Denoting the sequence $\xi^{l}$ shifted one step to the left as $T^{+} \vec{\xi}=\left\{\xi^{l+1}\right\}$ and, similarly the sequence shifted one step to the right $T \vec{\xi}=\left\{\xi^{l-1}\right\}$, we represent the above boundary conditions respectively as:

$$
\begin{aligned}
& \vec{\xi}_{-}+\left[b^{+} T^{+}+b_{0}+b T\right] \vec{\xi}_{+}=0 \equiv \vec{\xi}_{-}+B \vec{\xi}_{+}, \\
& \vec{\xi}_{+}+\left[c^{+} T^{+}+c_{0}+c T\right] \vec{\xi}_{+}=0 \equiv \vec{\xi}_{+}+C \vec{\xi}_{-},
\end{aligned}
$$

Regarding the periodicity of the chain of quantum dots, the corresponding sequences of their Weyl-Titchmatsh or Krein finctions $\operatorname{diag} \mathcal{M} \equiv \oplus \sum \mathcal{M}_{l}$ are also periodics:

$$
T^{+} \operatorname{diag} \mathcal{M} T=T \operatorname{diag} \mathcal{M} T^{+}=\operatorname{diag} \mathcal{M}
$$

With 1D contact subspaces $E_{l} \equiv E$ and the interaction $B$ or $C$ between the boundary data on the chain, the boundary values of the relevant Bloch functions $\vec{\Xi}$ are quasi-periodic $\Xi_{ \pm}^{l}=e^{i p l} \Xi_{ \pm}^{0}$, with regard to the quasimomentum $p:$

$$
\begin{gathered}
\mathcal{M} \Xi_{+}^{0}-\left[b^{+} e^{-i p}+b_{0}+b e^{i p}\right] \Xi_{+}^{0}=0, \text { for the boundary codition } B \text {, and } \\
\Xi_{+}^{0}-\mathcal{M}\left[c^{+} e^{-i p}+c_{0}+c e^{i p}\right] \Xi_{+}^{0}=0
\end{gathered}
$$

for the boundary condition $C$. In the case of the quantum dot substituted by the zero-range boundary condition with an inner structure, as in Appendix 1:

$$
\mathcal{M}=P_{E} \frac{I+\lambda A}{A-\lambda I} P_{E}=-P_{E} A P_{E}+P_{E} \frac{I+A^{2}}{A-\lambda I} P_{E} \approx-P_{E} A P_{E}-\frac{P_{E}\left(I+A^{2}\right) P_{E}}{\lambda}+O(1 / \lambda)^{2}
$$

For a special choice of the boundary parameters $-P_{E} A P_{E}=b_{0}$ with regards to $\mathcal{M}+b_{0} \rightarrow 0$ or, correspondingly, $c_{0} P_{E} A P_{E}=1$ with regards to $I+\mathcal{M} c_{0} \rightarrow 0$ while $\lambda \rightarrow \infty$, we get for the special choice of the boundary parameters:

and correspondingly:

$$
P_{A} \frac{I+A^{2}}{A-\lambda} P_{E} \Xi_{+}^{0}+2|b| \cos \left(p+\varphi_{b}\right) \Xi_{+}^{0}=0
$$

$$
\left(I+c_{0} P_{E} A P_{E}\right) \Xi_{+}^{0}-P_{E} \frac{\left(I+A^{2}\right)}{A-\lambda I} P_{E} 2|c| \cos \left(p+\varphi_{c}\right) \Xi_{+}^{0}=0 .
$$

The Nevanlinna function $P_{A} \frac{\left(I+A^{2}\right)}{A-\lambda} P_{E}$ is invertible on real axis, and the innverse is also a Nevanlinna function arising from a pair $E, G$ with a finite-dimensional operator $G: K \rightarrow K$ with selected deficiency subspace $N_{i}=E$

$$
-\left[P_{E} \frac{\left(I+A^{2}\right)}{A-\lambda} P_{E}\right]^{-1}=\lambda\left[P_{E}\left(I+A^{2}\right) P_{E}\right]^{-1}-P_{E} G P_{E}+P_{E} \frac{I+G^{2}}{G-\lambda I} P_{E},
$$

hence first addendum in (59) multiplied by $\left[P_{E} \frac{\left(I+A^{2}\right)}{A-\lambda} P_{E}\right]^{-1}$ yields:

$$
\left(I+c_{0} P_{E} A P_{E}\right)\left[\lambda\left[P_{A}\left(I+A^{2}\right) P_{E}\right]^{-1} \Xi_{+}^{0}-P_{E} G P_{E}+P_{E} \frac{I+G^{2}}{G-\lambda I} P_{E}\right] \Xi_{+}^{0} .
$$

\footnotetext{
${ }^{4}$ The operators $A$ and $G$ are connected similarly to Laplacean with Dirichlet and Neumann boundary conditions on a domain with a smooth boundary.
} 
This allows one to rewrite the dispersion equation (59) as:

$$
\left(I+c_{0} P_{E} A P_{E}\right)\left[\lambda\left[P_{A}\left(I+A^{2}\right) P_{E}\right]^{-1} \Xi_{+}^{0}-P_{E} G P_{E}+P_{E} \frac{I+G^{2}}{G-\lambda I} P_{E}\right] \Xi_{+}^{0}+2|c| \cos \left(p+\varphi_{c}\right) \Xi_{+}^{0}=0 .
$$

The equations $(58,62)$ show typical dispersion functions of $1 \mathrm{D}$ periodic lattices.

The most important characteristics of periodic lattices are the quasimomentum and the effective mass, which are calculated from the dispersion function as the inverse of the derivative of energy with respect to the quasimomentum $\left[\frac{\partial \lambda}{\partial p_{s} \partial_{t}}\right]^{-1} \equiv m_{s t}$. For a 1D discrete periodic chain of "quantum dots", we calculate the effective mass in terms of the relevant Krein function ( abstract analog of the Weyl-Titchmarsh function) and the boundary parameter $\beta$.

Assuming that the dispersion equation for the chain is $\mathcal{M}-b \cos p=0$, we differentiate the dispersion equation twice with respect to quazimomentum:

$$
\begin{gathered}
\mathcal{M}(\lambda)-\beta \cos p=0 \longrightarrow \frac{d \mathcal{M}}{d \lambda} \frac{d \lambda}{d p}+\beta \sin p=0 \longrightarrow \\
\longrightarrow \frac{d^{2} \mathcal{M}}{d \lambda^{2}}\left[\frac{d \lambda}{d p}\right]^{2}+\frac{d \mathcal{M}}{+} d \lambda \frac{d^{2} \lambda}{d p^{2}}+\beta \cos p=0,
\end{gathered}
$$

and use the 1D the 1D formula for the effective mass $m=\left[\frac{d^{2} \lambda}{d p^{2}}\right]^{-1}$. This implies:

$$
m=-\frac{\frac{d \mathcal{M}}{d \lambda}}{\beta \cos p+\frac{d^{2} \mathcal{M}}{d \lambda^{2}}\left(\frac{d \lambda}{d p}\right)^{2}}
$$

In experiment, the effective mass is usually measured at the ends of the spectral bands $\sin p=0$, where $\frac{d \lambda}{d p}=0$. Then the second term in the denominator vanishes and we get at the ends of spectral bands:

$$
m=-\frac{\frac{d \mathcal{M}}{d \lambda}}{\beta \cos p}=-\frac{\frac{d \mathcal{M}}{d \lambda}}{\mathcal{M}(\lambda)}
$$

\section{Spectral structure of a 1D superlattice via analytic perturbation procedure}

We consider a couple of two non-enteracting 1D discrete periodic lattices with typical dispersion equations similar to above $(58,62)$ and the corresponding weakly perturbed pair:

$$
\begin{gathered}
-\frac{A_{1}}{\lambda} e_{1}+B_{1} \lambda e_{1}+C_{1} e_{1}-\beta_{1} \cos p, \quad e_{1}=0, \quad \frac{2 A_{2} \lambda}{1-\lambda^{2}} e_{2}-\beta_{2} \cos p e_{2}=0 \\
-\frac{A_{1}}{\lambda} e_{1}+B_{1} \lambda e_{1}+C_{1} e_{1}-\cos p\left[\beta_{1} e_{1}+\delta e_{2}\right]=0, \frac{2 A_{2} \lambda}{1-\lambda^{2}} e_{2}-\cos p\left[\beta_{2} e_{2}+\delta e_{1}\right]=0 .
\end{gathered}
$$

Hereafter we assume, with regard of the diagram (10) that $B_{1}>0, A_{1,2}>0$ are small, and $\delta_{1,2}>0$ are much smaller and $\beta_{1,2}>0$. The perturbed dispersion equation is reduced to the determinant condition for the above linear system:

$$
\operatorname{det}\left(\begin{array}{cc}
-\frac{A_{1}}{\lambda}+B_{1} \lambda+C_{1}-\cos p \beta_{1} & \delta \cos p \\
\delta \cos p & \frac{2 A_{2} \lambda}{1-\lambda^{2}}-\cos p \beta_{2}
\end{array}\right)=0 .
$$

The perturbed spectral bands are found from the determinant condition with regard of real quasimomentum $p:-1<\cos p<1$. The determinant condition is presented as:

which implies:

$$
\begin{gathered}
{\left[-\frac{2 A_{1} A_{2}}{1-\lambda^{2}}-\frac{2 A_{2} B_{1} \lambda^{2}}{1-r \lambda^{2}}\right]+\cos p\left[\left(\frac{A_{1}}{\lambda}-B_{1} \lambda\right) \beta_{2}-\beta_{1} \frac{2 A_{2} \lambda}{1-\lambda^{2}}\right]+} \\
+\left(\beta_{1} \beta_{2}-\delta^{2}\right) \cos ^{2} p \equiv-K_{0}-\cos p K_{1}+K_{2} \cos ^{2} p=0
\end{gathered}
$$

$$
\left|K_{0} \pm \sqrt{K_{1}^{2}+4 K_{0} K_{2}}\right| \leq 2 K_{0}
$$

The ultimate condition corresponds, depending on the choice of parameter, to various physical conditions and the corresponding different physical properties of the perturbed superlattice. 


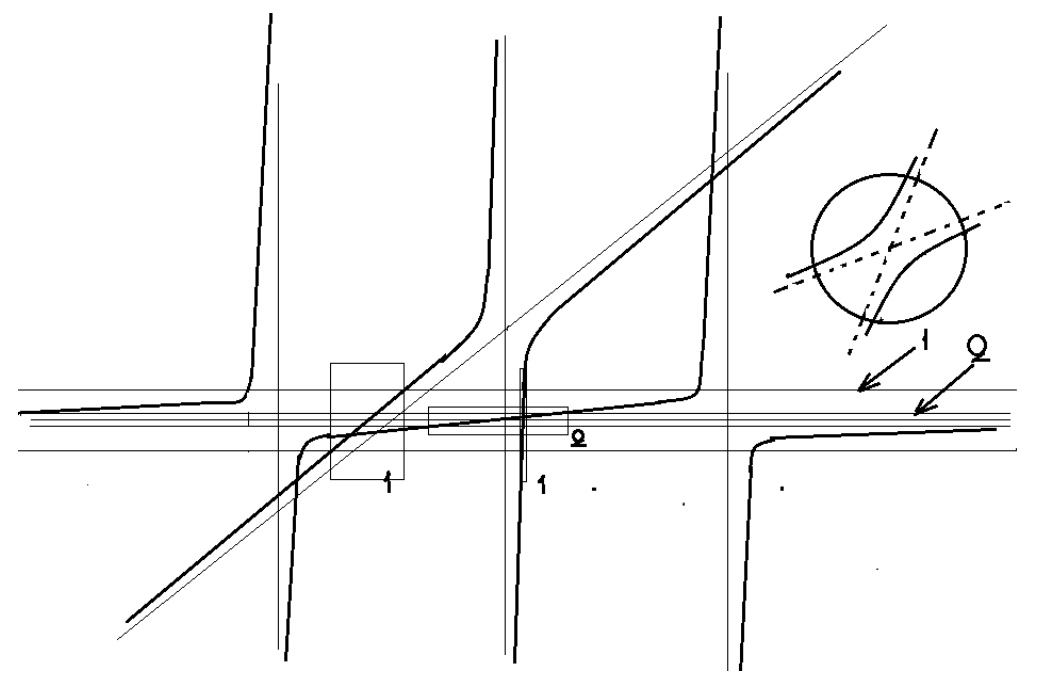

FIG. 10. The spectral structure of the perturbed superlattice with regards to quasi-crossings of terms of the underlying unperturbed lattices. The typical quasi-crossing for terms of the Nevanlinna-class dispersion equations. The unperturbed spetral bands are shown as thin rectangles marked by numbers 1,2

\section{Acnowledgements}

The autors are grateful to V. B. Bogevolnov for providing extremely interesting and inspiring material - the carbon flakes obtained by detonation - which served main object of our experimental and the theoretical study. We are also grateful to Prof. G. Fursey, Prof. P. Konorov, Prof. P. Schwerdtfegger and Dr. Krista Steenbergen for an inspirating discussion of the mathematical analog of the shaky concept of chemical bound with respect to the problem of selection appropriate contact subspaces for fitted solvable models of periodic lattices.

\section{References}

[1] Fursey G.N. Field emission in vacuum microelectronics. Kluwer Academic/Plenum Publishers (now Springer), New York, 2005.

[2] Fursey G., Konorov P., Pavlov B. and Adil Yafyasov. Dimensional Quantization and the Resonance Concept of the Low-Threshold Field Emission, Electronics, 2015, 4, P. 1101-1108.

[3] Yafyasov A., Bogevolnov V., Fursey G., Pavlov B., Polyakov M., Ibragimov A. Low-threshold field emission from carbon nano-clusters. Ultramicroscopy, 2011, 111, P. 409-414.

[4] Bonard J-M., Stöckli T., Majer F., W. de Heer, Chtelain A., Salvetat J-P., Forro L. Field-emission-Induced luminiscence from Carbon Nanotubes. Phys. Rev. Letters, 81, 7-17 August 1998, P. 1441-1444.

[5] Modinos A., Xanthakis J.P. Electron emission from amorphous carbon nitride film. Applied Physics Letters, Sept. 1998, 73,(13), P. 28.

[6] Forbes G., Xanthakis J.P. Field penetration into amorphous-carbon films: consequences for field-induced electron emission. Surf. Interface Anal.,2007, 39, P. 139-145.

[7] Fursey G., Polyakov M., Pavlov B., Yafyasov A., Bogevolnov V. Exceptionally low Threshold of Field Emission from carbon nanoclusters. Extended abstract. 24 International Vacuum Nanoelectronics conference. Bergishe Universitat, Wuppertal, Germany, 18-22 July 2011.

[8] Flugge S. Practical Quantum Mechanics I, Ch.2. Springer Verlag, Heidelberg, new York, 1974, xvi, 331 p.

[9] Datta S., Das Sarma B. Electronic analog of the electro-optic modulator. Appl. Phys. Lett., 1990, 56(7), P. 665-667.

[10] Shelykh I.A., Galki N.G.n, Bagraev N.T. Quantum splitter controlled by Rashba spin-orbit coupling. Phys. Rev.B, 2005 , 72, P. 235316.

[11] Harmer M., Pavlov B., Yafyasov A. Boundary condition at the junction. Journal of computational electronics, 2007,6 , P. $153-157$.

[12] Pavlov B. The theory of extensions and explicitly solvable models. (In Russian) Uspekhi Mat. Nauk, 1987, 42, P. 99-131.

[13] Pavlov B. A solvable model for scattering on a junction and a modified analytic perturbation procedure. Operator theory: Advances and Applications, 2009, 197, P. 281-335. Birkhäuser, Basel/Switzerland.

[14] Bagraev N.T. Metastable surface defects in p-type Ga As. Materials Science Forum vols, 1994, 143-147, P. $543-548$.

[15] Davies J.H. The Physics of Low-Dimensional Semiconductor. An Introduction, Cambridge University. Press: Cambridge, UK, 1998 , P. 438.

[16] Fursey G.N., Egorov N.V., Zakirov I.I., et al. Peculiarities of the total energy disribution of the field emission electrons from graphene-like structures: Distribution on full energy. Radiotechnika i electronika., 2015, 6,11, P. 1-4. (in press) (In Russian).

[17] Karpeshina Yu.E. Spectrum and Eigenfunctions of the Schrödinger operator in a three-dimensional space with periodic point potential of two-dimensional lattice type. In Russian $T M F, 1983$, 57(3), P. 414-423.

[18] von Neumann J. Mathematical foundations of quantum mechanics. Twelfth printing. Princeton Landmarks in Mathematics. Princeton Paperbacks. Princeton University Press, Princeton, NJ, 1996.

[19] Fermi E. Sul motto del neutroni nelle sostanze idrigenante. Ricerca Sci., 1936, P. 13-52.

[20] Berezin F.A.,Faddeev L.D. A remark on the Schrödinger equation with with a singular potential. Soviet Math. Doklady, 1961 , 2, P. 372-376. 
[21] Albeverio S. and Kurasov P. Singular Perturbations of Differential Operators. Solvable Schrödinger Type Operators. London Mathematical Society Lecture Note Series vol 271, Cambridge University Press, 2000, xiv+429 p.

[22] Bagraev N., Mikhailova A., Pavlov B., Prokhorov L., Yafyasov A. Parametric regimes of a resonance quantum switch. Phys. Rev. B, 2005, 71, P. 165308.

[23] Pavlov B., Antoniou I. Jump-start in the analytic perturbation procedure for the Friedrichs model. J. Phys. A (Math. Gen), 2005, 38, P. $4811-4823$.

[24] Pavlov B. A star-graph model via operator extension. Mathematical Proceedings of the Cambridge Philosophical Society, 2007, 142(02), P. 365-384.

[25] Krasnoselskij M.A. On selfadjoint extensions of Hermitian Operators. (in Russian) Ukrainskij Mat.Journal, 1949, 1(21), P. 21-38.

[26] S.P. Novikov private communication 2012, MIAN.

[27] Prigogine I. Irreversibility as a symmetry-breacking process. Nature, 1976, 246, P. 9.

[28] Martin G., Pavlov B., Yafyasov A. Resonance one-body scattering on a junction. Nanosystems: Physics, Chemistry, Mathematics, 2010, 1(1), P. 108-147.

[29] Pavlov B. S-Matrix and Dirichlet-to-Neumann Operators. Scattering and Semigroup Theory. In: Scattering, ed. R.Pike, P. Sabatier, Academic Press, Harcourt Science and Tech. Company. 2000, P. 1678-1688. 